\title{
Numerical Experiments with Monte Carlo Methods and SPAI Preconditioner for Solving System of Linear Equations
}

\author{
Bo Liu, Behrouz Fathi, and Vassil Alexandrov \\ Computer Science, \\ The University of Reading, \\ Whiteknight P.O. Box 225, \\ Reading, UK \\ $\{$ b.liu, fathi, v.n.alexandrov\}@rdg.ac.uk
}

\begin{abstract}
In this paper we present the results of experiments comparing the performance of the mixed Monte Carlo algorithms and SPAI preconditener with BICGSTAB. The experiments are carried out on a Silicon Graphics ONYX2 machine. Based on our experiments, we conclude that these techniques are comparable from the point of view of robustness and rates of convergence, with the Monte Carlo approach performing better for some general cases and SPAI approach performing better in case of very sparse matrices.
\end{abstract}

Keywords: Sparse Linear Systems, Preconditioned iterative methods, approximate inverse, SPAI, Monte Carlo methods.

\section{Introduction}

Consider a system of linear algebraic equations (SLAE) presented in the form:

$$
A x=b
$$

where $A$ is a real square $n \times n$ matrix, $x=\left(x_{1}, x_{2}, \ldots, x_{n}\right)^{t}$ is a $1 \times n$ solution vector and $b=\left(b_{1}, b_{2}, \ldots, b_{n}\right)^{t}$.

There are now quite a few deterministic methods based on Krylov subspace methods for solving general linear systems such as GMRES, BI-CGSTAB and QMR. In order to be effective these methods must be combined with a good preconditioner, and it is generally agreed that the choice of the preconditioner is even more crucial than the choice of the Krylov subspace iteration. The search for effective preconditioners is an active research topic in scientific computing. Several potentially successful methods have been proposed in the literature, and the SPAI preconditioner is regard as one of the most promising approaches [46]. However Monte Carlo methods are coming to play a role in finding a rough approximate inverse and few efficient parallel Monte Carlo approaches for MI have been presented in the past few years $[7,1]$. Nowadays, parallel Monte Carlo 
methods can be one of the most promising approaches for solving SLAE and also be an efficient preconditioner. We tested the algorithms solving general systems of linear equations, where the corresponding matrices were generated by simple generator constructing general dense and sparse matrices, which were stored in a row-packed format. Some matrices from the matrix market have also been used. We used Silicon Graphics ONYX2 machine to carry out the experiments.

The ideas of Monte Carlo for Matrix Computation are presented in Section 2, the main algorithm is described in Section 3 and the parallel approach and some numerical experiments are presented in Section 4.

\section{Monte Carlo for Matrix Computation}

Let us assume that we need to solve a general SLAE in form (1). Consider the general case when $\|I-A\| \geq 1$. We consider the splitting $A=B_{n}-B_{1}$, where the off-diagonal elements of $B_{n}$ are the same as $A$. The diagonal elements of $B_{n}$ are defined as $b_{i i}=a_{i i}+\gamma_{i}\|A\|$, choosing in most cases $\gamma_{i}>1, i=1,2, \ldots, n$. We then split $B_{n}=M-K$, where $M$ is diagonal matrix of $B_{n}$. We could transform system (1) to

$$
x=C x+f
$$

where $C=B_{n}^{-1} B_{1}$ and $f=B_{n}^{-1} b$. The multipliers $\gamma_{i}$ are chosen so that they reduce the norm of $C$ and reduce the number of Markov chains required to reach a given precision, in a similar way as proposed in [3]. Therefore we consider two possibilities, first, finding the solution of $x=C x+f$ using Monte Carlo (MC) method and, second, finding $A^{-1}$ and obtaining the solution via $x=A^{-1} f$. In this paper we are more interested in the second idea as a general goal, where we compute the approximate inverse of $B_{n}$ with $\mathrm{MC}$, then retrieve $A^{-1}$ via a function that will be introduced in the next section.

Consider firstly the stochastic approach. Assume that the system is transformed to its iterative form (2). Consider now the Markov Chain given by:

$$
s_{0} \rightarrow s_{1} \rightarrow \cdots \rightarrow s_{k}
$$

where the $s_{i}, i=1,2, \cdots, k$, belongs to the state space $S=\{1,2, \cdots, n\}$. Then for $\alpha, \beta \in S, p_{0}(\alpha)=p\left(s_{0}=\alpha\right)$ is the probability that the Markov chain starts at state $\alpha$ and $p\left(s_{j+1}=\beta \mid s_{j}=\alpha\right)=p_{\alpha \beta}$ is the transition probability from state $\alpha$ to state $\beta$. The set of all probabilities $p_{\alpha \beta}$ defines a transition probability matrix $P=\left\{p_{\alpha \beta}\right\}_{\alpha, \beta=1}^{n}[7]$ [8]. We say that the distribution $\left(p_{1}, \cdots, p_{n}\right)^{t}$ is acceptable for a given vector $g$, and that the distribution $p_{\alpha \beta}$ is acceptable for matrix $C$, if $p_{\alpha}>0$ when $g_{\alpha} \neq 0$, and $p_{\alpha} \geq 0$, when $g_{\alpha}=0$, and $p_{\alpha \beta}>0$ when $C_{\alpha \beta} \neq 0$, and $p_{\alpha \beta} \geq 0$ when $C_{\alpha \beta}=0$ respectively. We assume $\sum_{\beta=1}^{n} p_{\alpha \beta}=1$, for all $\alpha=1,2, \cdots, n$. Generally, we define

$$
W_{0}=1, W_{j}=W_{j-1} \frac{C_{s_{j-1} s_{j}}}{p_{s_{j-1} s_{j-1}}}
$$

for $j=1,2, \cdots, n$. 
Consider now the random variable $\theta[g]=\frac{g_{s_{0}}}{p_{s_{0}}} \sum_{i=1}^{\infty} W_{i} f_{s_{i}}$. We use the following notation for the partial sum:

$$
\theta_{i}[g]=\frac{g_{s_{0}}}{p_{s_{0}}} \sum_{j=0}^{i} W_{j} f_{s_{j}} .
$$

Under the condition $\|C\|<1$, the corresponding Neumann series converges for any given $f$, and $E \theta_{i}[g]$ tends to $(g, x)$ as $i \rightarrow \infty$. Thus, $\theta_{i}[g]$ can be considered as an estimate of $(g, x)$ for $i$ sufficiently large. To find an arbitrary component of the solution, for example, the $r^{t h}$ component of $x$, we should choose,

$$
\begin{gathered}
g=e(r)=(\underbrace{0, \ldots, 1}_{r}, 0, \ldots, 0) \text { such that } \\
e(r)_{\alpha}=\delta_{r \alpha}= \begin{cases}1 & \text { if } r=\alpha \\
0 & \text { otherwise }\end{cases}
\end{gathered}
$$

It follows that

$$
(g, x)=\sum_{\alpha=1}^{n} e(r)_{\alpha} x_{\alpha}=x_{r} .
$$

The corresponding Monte Carlo method is given by

$$
x_{r}=\hat{\Theta}=\frac{1}{N} \sum_{s=1}^{N} \theta_{i}[e(r)]_{s}
$$

where $N$ is the number of chains and $\theta_{i}[e(r)]_{s}$ is the approximate value of $x_{r}$ in the $s^{t h}$ chain. It means that using Monte Carlo method, we can estimate only one, few or all elements of the solution vector. We consider Monte Carlo with uniform transition probability (UM) $p_{\alpha \beta}=\frac{1}{n}$ and Almost optimal Monte Carlo method (MAO) with $p_{\alpha \beta}=\frac{\left|C_{\alpha \beta}\right|}{\sum_{\beta=1}^{n}\left|C_{\alpha \beta}\right|}$, where $\alpha, \beta=1,2, \ldots, n$. Monte Carlo MI is obtained in a similar way $[7,1]$.

To find the approximate inverse $Q^{-1}=\left\{q_{r r^{\prime}}\right\}_{r, r^{\prime}=1}^{n}$ of some matrix $T$, we must first compute matrix

$$
F=I-T,
$$

where $I$ is the identity matrix. Clearly, the inverse matrix is given by

$$
Q=\sum_{i=0}^{\infty} F^{i}
$$

which converges if $\|F\|<1$.

To estimate the element $q_{r r^{\prime}}$ of the inverse matrix $Q$, we let the vector $f$ be the following unit vector

$$
f_{r^{\prime}}=e\left(r^{\prime}\right) .
$$


We then can use the following Monte Carlo method for calculating elements of the inverse matrix $Q$ :

$$
q_{r r^{\prime}} \approx \frac{1}{N} \sum_{s=1}^{N}\left[\sum_{\left(j \mid s_{j}=r^{\prime}\right)} W_{j}\right],
$$

where $\left(j \mid s_{j}=r^{\prime}\right)$ means that only

$$
W_{j}=\frac{F_{r s_{1}} F_{s_{1} s_{2}} \ldots F_{s_{j-1} s_{j}}}{p_{r s_{1}} p_{s_{1} s_{2}} \ldots p_{s_{j-1} s_{j}}}
$$

for which $s_{j}=r^{\prime}$ are included in the sum (11).

Since $W_{j}$ is included only in the corresponding sum of $r^{\prime}=1,2, \ldots, n$, the same set of $N$ chains can be used to compute a single row of the inverse matrix, which is one of the inherent properties of MC making them suitable for parallelization.

The probable error of the method, is defined as $r_{N}=0.6745 \sqrt{D \theta / N}$, where $P\left\{|\bar{\theta}-E(\theta)|<r_{N}\right\} \approx 1 / 2 \approx P\left\{|\bar{\theta}-E(\theta)|>r_{N}\right\}$, if we have $N$ independent realizations of random variable (r.v.) $\theta$ with mathematical expectation $E \theta$ and average $\bar{\theta}[8]$.

\section{The parallel approximate methods}

It is well-known that Monte Carlo (MC) methods are efficient for solving systems of linear algebraic equations. Initially MC methods have been applied to SLAEs with $\|I-A\|<1$. In the further developments [7,3,2] MC methods have been applied to solve SLAE with diagonally dominant and M-matrices. To use Monte Carlo algorithms for solving general systems of linear equations, we developed a new mixed algorithm, where for the system (1), firstly, we construct a diagonal dominant matrix $B_{n}$ whose off-diagonal elements are the same as those of $A$, then, secondly we compute the approximate inverse matrix of $B_{n}[3]$ using the algorithm below.

1. Initial data: Input matrix $B_{n}$, parameters $\delta$ and $\epsilon$.

2. Preprocessing:

2.1 Split $B_{n}=M-K$ where $\mathrm{M}$ is a diagonal matrix $m_{i i}=b_{i i} i=1,2, \ldots, n$.

2.2 Compute the matrix $C=M^{-1} K$.

2.3 Compute $\|C\|$ and the Number of Markov Chains $N=\left(\frac{0.6745}{\epsilon} \cdot \frac{1}{(1-\|C\|}\right)^{2}$.

3. For $\mathrm{i}=1$ to $n$;

3.1 For $j=1$ to $j=N$;

Markov Chain Monte Carlo Computation:

3.1.1 Set $t_{k}=0$ (stopping rule), $W_{0}=1, S U M[i]=0$ and Point $=i$.

3.1.2 Generate an uniformly distributed random number giving nextpoint.

3.1.3 If $C[$ point $][$ netxpoint $] !=0$.

LOOP 
3.1.3.1 Compute $W_{j}=W_{j-1} \frac{C[\text { point }][\text { netxpoint }]}{P[\text { point }][\text { netxpoint }]}$.

3.1.3.2 Set Point $=$ nextpoint and $S U M[i]=S U M[i]+W_{j}$.

3.1.3.3 If $\left|W_{j}\right|<\delta, t_{k}=t_{k}+1$

3.1.3.4 If $t_{k} \geq n$, end LOOP.

3.1.4 End If

3.1.5 Else go to step 3.1.2.

3.2 End of loop j.

3.3 Compute the average of results.

4. End of loop i.

5. Obtain The matrix $Z=(I-C)^{-1}$. then the expecting approximate matrix $Y_{n}=Z \times M^{-1}$.

Finally we retrieve the inversion matrix of $A$ via the following function [2]:

$$
Y_{n-1}=Y_{n}+\frac{Y_{n} S_{n} Y_{n}}{1-\operatorname{trace}\left(Y_{n} S_{n}\right)},
$$

where $Y_{n}$ is the computed approximate inverse of $\mathrm{B}, S_{n}=B_{n}-\operatorname{diag}(A), \mathrm{n}$ is a sequence of integers ranging from one to the size of the matrix $A$. It is obvious that the approximate solution vector can be obtained by $A^{-1} * b$. Indeed, before we retrieve $A^{-1}$ we also can apply the following refinement function

$$
Y_{n}^{\text {new }}=Y_{n}^{\text {old }}\left(2 \times I-Y_{n}^{\text {old }} B_{n}\right),
$$

where I denotes the identity matrix. Clearly the precision of the approximate solution vector can be controlled using the refinement function with just 1 or 2 steps. From the experimental work is evident that the number of steps required to obtain almost exact solution is at most 3 or 4 .

On the other hand we studied the SPAI preconditioner, which is based on the idea of Frobenius norm minimization. The approximate inverse matrix of $A$ is computed as matrix $M$ which minimizes $\|I-M A\|$ (or $\|I-A M\|$ for the right preconditioning) subject to some sparsity constraints, see $[4,5]$. Once the approximate inverse matrix of $A$ is known, SPAI applies BICGSTAB to solve (1) ( see http://www.sam.math.ethz.ch/grote/spai/)

Now we have introduced both algorithms in brief. We will use both of them to solve general SLAEs.

\section{Parallel Implementation and Numerical Results}

The Monte Carlo code is written in C using PVM and the SPAI code is written in $\mathrm{C}$ using MPI. To test the two approaches we consider matrices from the Matrix Market (http://math.nist.gov/MatrixMarket/) and some general matrices randomly generated by the generator we provide. The generator generates random numbers for the matrices with predefined sparsity. The experiments are carried out on a Silicon Graphics ONYX2 parallel machine with 12 processors (four 195

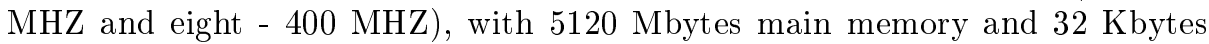


cache in total. We run both algorithms with the corresponding parameters. The computing time of the Monte Carlo calculation and the SPAI preconditioner are shown separately. The full time required to solve the SLAEs for mixed algorithms and SPAI with BICGSTAB is also shown. Some of the test matrices used in this experiment are given below.

- BWM200.MTX size $n=200$, with $n z=796$ nonzero elements, from Matrix Market web site;

- CAVITY03.MTX size $\mathrm{n}=327$, with $\mathrm{nz}=7327$ nonzero elements, from Matrix Market web site;

- GEN200_DENSE.MC size $\mathrm{n}=200$, dense, from the generator;

- GEN200_60SPAR.MC size $\mathrm{n}=200$, with $\mathrm{nz}=16000$ nonzero elements, from the generator;

- GEN800_DENSE.MC size $\mathrm{n}=800$, dense, from the generator;

- GEN800_70SPAR.MC size $\mathrm{n}=800$, with $\mathrm{nz}=192000$ nonzero elements, from the generator;

- GEN2000_99SPAR.MC size $n=2000$, with $n z=40000$ nonzero elements, from the generator;

- GEN500_90SPAR.MC size $\mathrm{n}=500$, with $\mathrm{nz}=25000$ nonzero elements, from the generator;

- GEN1000_95SPAR.MC size $\mathrm{n}=1000$, with $\mathrm{nz}=50000$ nonzero elements, from the generator;

The default parameters we set up in Monte Carlo methods are

1. $\epsilon=0.05$ denotes a given stochastic error;

2. $\delta=0.01$ denotes the accuracy of Monte Carlo approximation;

3. step $=1$ denotes how many steps are spent on the refinement function, in each single step of using the refinement function two matrix-by-matrix multiplications are computed.

We apply the appropriate parameters in SPAI and show the best performance with the best combination of these parameters. R-MC denotes the residual computing time of Monte Carlo approach which includes the time for the refinement procedure, the retrieval procedure and obtaining the approximate solution vector. MC denotes the time required for Monte Carlo algorithm only. Therefore, TOTAL-MC is the time required for the MC and R-MC. R-SPAI denotes the residual computing time of SPAI, which includes the time of BICGSTAB for obtaining the approximate solution vector (the block procedure and scalar-matrix procedure while using block algorithm). SPAI denotes the time required by the SPAI preconditioner. TOTAL-SPAI is the total time of SPAI and R-SPAI. ERROR denotes the absolute error of the approximate solution vector given below.

$$
\text { ERROR }=\frac{\left\|x_{\text {exact }}-x_{\text {approximate }}\right\|_{2}}{\left\|x_{\text {exact }}\right\|_{2}}
$$




\begin{tabular}{|c|c|c|c|c|c|c|c|}
\hline Para & $\overline{\mathrm{BL}}$ & SPAI & R-SPAI & TOTAL-SPAI & $\overline{\mathrm{MC}}$ & R-MC & TOTAL-MC \\
\hline 0.5 & $1 \times 1$ & 0.9303448 & 85.6012096 & 86.5315544 & \multirow{6}{*}{2.186874} & \multirow{6}{*}{3.164163} & \multirow{6}{*}{5.351037} \\
\hline & $3 \times 3$ & 0.9759936 & 75.2054016 & 76.1813952 & & & \\
\hline \multirow[t]{2}{*}{0.6} & $1 \times 1$ & 0.8856872 & 220.1436288 & 221.029316 & & & \\
\hline & $3 \times 3$ & 0.9230960 & 150.3301808 & 151.2532768 & & & \\
\hline \multirow[t]{2}{*}{0.7} & $1 \times 1$ & 1.3930344 & 351.6003544 & 352.9933888 & & & \\
\hline & $3 \times 3$ & 1.1148200 & 160.762056 & 161.876876 & & & \\
\hline
\end{tabular}

Table 1. BWM200.MTX Solution with parameters: $E R R O R=10^{-4}$ and $P=10$ processors. SPAI is tested with different values of $\epsilon$, and both blocked and non-blocked.

\begin{tabular}{|c|c|c|c|c|c|c|c|}
\hline Para & $\mathrm{BL}$ & SPAI & R-SPAI & TOTAL-SPAI & MC & R-MC & TOTAL-MC \\
\hline \multirow[t]{2}{*}{0.5} & $1 \times 1$ & 2.9910024 & nan & $\operatorname{nan}$ & \multirow{6}{*}{0.9338300} & \multirow{6}{*}{2.346462} & \multirow{6}{*}{3.280292} \\
\hline & $3 \times 3$ & 1.1972320 & nan & nan & & & \\
\hline \multirow[t]{2}{*}{0.6} & $1 \times 1$ & 3.1077280 & nan & nan & & & \\
\hline & $3 \times 3$ & 1.2565296 & nan & nan & & & \\
\hline \multirow[t]{2}{*}{0.7} & $1 \times 1$ & 2.6192136 & nan & nan & & & \\
\hline & $3 \times 3$ & 0.9718288 & nan & nan & & & \\
\hline
\end{tabular}

Table 2. GEN200_DENSE.MC Solution with parameters: ERROR $=10^{-4}$ and $\mathrm{P}=10$ processors. SPAI is tested with different values of $\epsilon$, and both blocked and nonblocked. 
SPAI is run with the default parameter of iterations in BICGSTAB, 500, and nan means we can not obtain the results under the setting, or it is converging slowly.

\begin{tabular}{||l|l|l|l|l||}
\hline \hline P\# & MC & TOTAL-MC & SPAI & TOTAL-SPAI \\
\hline 2 & 76.20574 & 85.813108 & 0.2917584 & nan \\
\hline 5 & 36.16877 & 60.095253 & 0.5471512 & nan \\
\hline 8 & 28.29067 & 46.830696 & 0.3889352 & nan \\
\hline 12 & 21.07290 & 33.248477 & 1.3224760 & nan \\
\hline \hline
\end{tabular}

Table 3. CAVITY03.MTX ERROR $=10^{-4}$ and $\mathrm{P}=2,5,8,12$ processors respectively.

\begin{tabular}{||l|l|l|l|l||}
\hline \hline P\# & MC & TOTAL-MC & SPAI & TOTAL-SPAI \\
\hline 2 & 156.1388 & 586.202544 & 130.6839168 & nan \\
\hline 5 & 47.70701 & 385.626890 & 67.6120952 & nan \\
\hline 8 & 36.74342 & 304.847875 & 24.0445288 & nan \\
\hline 12 & 26.53539 & 294.639845 & 23.1435808 & nan \\
\hline \hline
\end{tabular}

Table 4. GEN800_70SPAR.MC ERROR $=10^{-4}$ and $\mathrm{P}=2,5,8,12$ processors respectively.

\begin{tabular}{||l|l|l|l|l|}
\hline \hline Matrix & MC & TATOL-MC & SPAI & TOTAL-SPAI \\
\hline GEN200_60SPAR.MC & 1.047589 & 4.720657 & 1.1548728 & nan \\
\hline GEN500_90SPAR.MC & 7.730741 & 84.94246 & 4.1712408 & nan \\
\hline GEN800_DENSE.MC & 24.32269 & 300.3137 & 29.000558 & nan \\
\hline GEN1000_95SPAR.MC & 46.274798 & 746.9745 & 9.1504488 & nan \\
\hline GEN2000_99SPAR.MC & 302.626325 & 4649.0219003 & 6.2901104 & nan \\
\hline \hline
\end{tabular}

Table 5. ERROR $=10^{-4}$ and $\mathrm{P}=10$ processors

The examples show that for some cases MC converges much faster than SPAI. In case of some general matrices it can be seen that SPAI is converging 
very slowly. There are also cases where SPAI is performing much better than MC. Further experiments are required to carry out detailed analysis.

\section{Conclusion}

We have presented the results of experiments comparing the efficiency of parallel SPAI preconditioner with BICGSTAB and parallel mixed Monte Carlo algorithms. The experiments are carried out on a Silicon Graphics ONYX2 machine. Based on our experiments, we conclude that these techniques are comparable from the point of view of robustness and rates of convergence, with the Monte Carlo approach being somewhat better for some general cases and SPAI approach performing better in case of very sparse matrices. We can also conclude that both techniques offer excellent potential for use on high performance computers.

\section{References}

1. Alexandrov V.N., Efficient parallel Monte Carlo Methods for Matrix Computation, Mathematics and computers in Simulation, Elsevier 47 pp. 113-122, Netherlands, (1998).

2. Fathi Vajargah B., Liu B. and Alexandrov V.,Mixed Monte Carlo Parallel Algorithms for Matrix Computation, ICCS 2002, Amsterdam, Netherlands.

3. Fathi Vajargah B., Liu B. and Alexandrov V., On the preconditioner Monte Carlo methods for solving linear systems. MCM 2001, Salzburg, Austria (to appear).

4. M.Benze, C.D.Meyer, and M.Tuma, A sparse approximate inverse preconditioner for the conjugate gradient method, SIAM, 1996.

5. Marcus J.Grote, Thomas Huckle, Parallel Preconditioning with Sparse Approximate Inverses, SIAM J. of Scient. Compute. 18(3), 1997.

6. S.T. Barnard and M. J. Grote, in Proc. A Block Version of the SPAI Preconditioner, 9th SIAM Conf. on Parallel. Process. for Sci. Comp., held March 1999.

7. Dimov I., Alexandrov V.N. and Karaivanova A., Resolvent Monte Carlo Methods for Linear Algebra Problems, Mathematics and Computers in Simulation, Vo155, pp. 25-36, 2001.

8. Sobol I.M. Monte Carlo numerical methods. Moscow, Nauka, 1973 (in Russian). 